\title{
SIMPLE AND COMPLEX DYNAMICS FOR CIRCLE MAPS*
}

\author{
Lluís Alsedà and Vladimir Fedorenko
}

\begin{abstract}
The continuous self maps of a closed interval of the real line with zero topological entropy can be characterized in terms of the dynamics of the map on its chain recurrent set. In this paper we extend this characterization to continuous self maps of the circle. We show that, for these maps, the chain recurrent sel can exhibit a new dynamic behaviour which is specific of the circle maps of degree one.
\end{abstract}

\section{Introduction.}

The aim of this paper is to extend the characterization of the complex and simple interval maps (in the sense of positive or zero topological entropy respectively) to circle maps. We shall start by stating this characterization for interval maps for completeness (see Theorem A). To do it we have to introduce the appropriate notation.

Let $f$ be a map from a topological space $X$ into itself. We shall denote by $f^{n}$ the map $f \circ f \circ \ldots \circ f n$ times (if $n=0$ we set $f^{n}=$ Id).

Let now $f$ be an interval map (that is, a continuous map from a closed interval $I$ of the real line into itself). We say that $f$ has a horseshoe if there exist $n>0$ and two closed intervals $I_{1}, I_{2} \subset I$ with pairwise disjoint interiors such that $I_{1} \cup I_{2} \subset f^{n}\left(I_{1}\right)$ and $I_{1} \cup I_{2} \subset f^{n}\left(I_{2}\right)$.

The above condition was used for the first time by Sharkovskir(see [13]) and has been used widely in the study of interval maps (see [9], [4] and [12]). The name of horseshoe was given to this condition by Misiurewicz

\footnotetext{
*This paper was written during a stage of the sccond author at the Centre de Recerca Matemàtica. He was also partially supported by the Fund for Fundamental Research of the State Committee of Ukraine on Science and Technology grant number 1/356. The first author has been partially supported by the DGICYT grant number PB900695 .
} 
in [9]. An interval map having a horseshoe was called turbulent by Block (see [4]) and in [12] a similar notion was called an $L$-scheme.

Wc note that the set of interval maps having a horseshoe is open and dense in the space of all interval maps (we suppose this space endowed with the topology of the uniform convergence). Thus, in this sense, the property that a map has a horseshoe is generic.

It is well known that a map has positive topological entropy if and only if it has a horseshoe (sce [9]). In other words, the existence of horseshoes characterizes the complex interval maps.

Now we introduce the necessary notions to characterize the simple interval maps. Let $S$ be a closed invariant set of an interval map $f$. We say that $S$ splits into $S_{0}$ and $S_{1}$ if $S_{0}$ and $S_{1}$ are closed nonempty subsets of $S$ such that $\left\langle S_{1}\right\rangle \cap\left\langle S_{2}\right\rangle=\emptyset$ (where $\left\langle S_{i}\right\rangle$ denotes the convex hull of $\left.S_{i}, i=1,2\right), S_{1} \cup S_{2}=S, f\left(S_{1}\right)=S_{2}$ and $f\left(S_{2}\right)=S_{1}$. We also say that $S$ splits $k$ times if $S$ splits into $S_{0}$ and $S_{1}$ and each of them splits $(k-1)$ times under $f^{2}$. The set $S$ is said to be simple if either it is a fixed point or it splits $k$ times for each $k \leq \log _{2}$ Card $S$ (sec [6]).

Remark 1.1. From the above definition it follows easily that each simple set cither consists on a unique periodic orbit or does not contain any periodic orbit.

Recall that if $f$ is a continuous map from a metric space $X$ into itself the set of chain recurrent points of $f$ is denoted by $C R(f)$ and is defined to be the set of all $x \in X$ such that for each $\varepsilon>0$ there exists $\left\{x_{i}\right\}_{i=0}^{n}$ with $x_{0}=x_{n}=x$ and $\left|f\left(x_{i}\right)-x_{i+1}\right|<\varepsilon$ for $i=0,1,2, \ldots, n-1$.

The following theorem characterizes the complex and simple interval maps (see $[6]$ ).

Theorem A. Each interval map $f$ satisfies one and only one of the following two conditions.

(a) f has a horseshoe.

(b) The chain recurrent set of $f$ is the union of all simple sets of $f$.

To cxtend Theorem A to circle maps we have to reformulate the above notions in this context.

We shall represent the circle $\mathrm{S}^{1}$ as the set $\{z \in \mathbb{C}:|z|=1\}$. Any continuous map from $\mathbf{S}^{1}$ into itself will be called a circle map.

We note that the notion of a simple set and of horseshoe extends naturally to circle maps by simply replacing closed intervals by closed arcs of the circle (that is, subsets of $\mathbf{S}^{1}$ which are homeomorphic to closed intervals of the real line). 
We note that if an interval map $f$ has a horseshoe $I_{1}, I_{2}$ then we always have that, for each $i, j \in\{1,2\}$, there exist a closed interval $I_{j}^{i} \subset I_{i}$ such that $f^{n}\left(I_{j}^{i}\right)=I_{j}$. However, this is not the case if we are talking about horseshoes of circle maps. Indeed, if $I_{1}, I_{2} \subset \mathbf{S}^{1}$ is a horseshoe of a circle map $g$ it may happen that $g^{n}\left(I_{1}\right)=\mathbf{S}^{1}$ in such a way that $\left.g^{n}\right|_{\operatorname{Int}\left(I_{1}\right)}$ is injective and $g^{n}(a)=g^{n}(b) \in \operatorname{Int}\left(I_{2}\right)$ where $a$ and $b$ denote the two endpoints of $I_{1}$. Then, clearly, does not hold that for each $i, j \in\{1,2\}$ there exist a closed arc $I_{j}^{i} \subset I_{i}$ such that $g^{n}\left(I_{j}^{i}\right)=I_{j}$.

Let $f$ be a map from a topological space $X$ into itself and let $x \in X$. We say that $x$ is a periodic point of $f$ if $f^{n}(x)=x$ for some $n>0$. The smallest $n$ with the above property is called the period of $x$. If $x \in X$ is a periodic point of $f$ of period $n$ then the set $\left\{x, f(x), \ldots, f^{n-1}(x)\right\}$ will be called a periodic orbit of $f$ of period $n$. In the sequel we shall denote by $\operatorname{Per}(f)$ the set of periods of all periodic points of $f$. Also, if $x \in X$ we shall denote by $\omega_{x}(f)$ the omega limit set of $x$ which is defined to be the set of all accumulation points of $\left\{f^{n}(x): n \geq 0\right\}$. We will also use the notation $w(f)$ to denote $\cup_{x \in X} \omega_{x}(f)$.

The main result of this paper is the following.

Theorem 1.2. Each circle map $f$ satisfies one and only one from the following three conditions:

(a) f has a horseshoe.

(b) There exist $n>0$ such that $\omega_{x}\left(f^{n}\right)$ is a simple set for each $x \in \mathbf{S}^{\mathbf{1}}$.

(c) $\operatorname{Per}(f)=\emptyset$.

We note that, as for interval maps, Condition (a) of the above theorem is generic in the space of circle maps endowed with the topology of the uniform convergence and is a criterion for positive topological entropy (see [9]). On the other hand, it is known that Condition (b) with $n=1$, in the case of an interval map, is equivalent to Condition (b) of Theorem $A$ (see for instance Theorem 2 of [7]). However, for circle maps it is not. Theorem 1.2 is stated in this way for simplicity but in Section 4 the topological picture of the chain recurrent set in this case will be described in detail. Finally, the dynamics of a circle map satisfying Condition (c) of the above theorem can be roughly described as follows. The map $f$ has a unique $\omega$-limit set which is minimal (i.e. it has no closed invariant proper subset) and the restriction of $f$ to it is semi-conjugate to a rotation of the circle by an irrational angle. A detailed description of the dynamics of such a map can be found in [3] and [11]. 


\section{Definitions and preliminary results.}

In this section we will introduce the necessary notation to prove Theorem 1.2. Also we will prove a lemma that will play a key role in that proof.

Let $f$ be a circle map. As usual, instead of working with $f$ itself we shall use a lifting of $f$. A continuous map $F: \mathbb{R} \rightarrow \mathbb{R}$ is called a lifting of $f$ if $e \circ F=f \circ e$, where $e(x)=\exp (2 \pi i x)$ is the natural projection from $\mathbb{R}$ to $\mathbf{S}^{1}$. We note that if $F$ is a lifting of $f$ then $F+m$ is also a lifting of $f$ for each $m \in \mathbb{Z}$ and that $F^{n}$ is a lifting of $f^{n}$. Also, there exists an integer $d$ such that $F(x+1)=F(x)+d$ for each $x \in \mathbb{R}$. This number $d$ is called the degree of $f$ and is denoted by $\operatorname{deg}(f)$. It is not difficult to see that $\operatorname{deg}\left(f^{n}\right)=\operatorname{deg}(f)^{n}$.

We say that a point $x \in \mathbb{R}$ is periodic (mod. 1) of period $q$ for $F$ if $F^{q}(x)-x \in \mathbb{Z}$ but $F^{j}(x)-x \notin \mathbb{Z}$ for $j=1,2, \ldots, q-1$. Clearly, $x$ is a periodic (mod. 1) point of $F$ of period $q$ if and only if $e(x)$ is a periodic point of $f$ of period $q$.

Let $F$ be a lifting of a circle map $f$. In the sequel we shall denote by $\operatorname{Per}(F)$ the set of periods of all periodic (mod. 1) points of $F$. Clearly, $\operatorname{Per}(f)=\operatorname{Per}(F)$

Let $f$ be a circle map of degree one and let $F$ be a lifting of $f$. For $x \in \mathbb{R}$ we define its $F$-rotation number as

$$
\limsup _{n \rightarrow \infty} \frac{F^{n}(x)-x}{n}
$$

and denote it by $\rho_{F}(x)$. We note that, since $f$ has degree $1, \rho_{F}(x)=$ $\rho_{F}(x+m)$ for all $m \in \mathbb{Z}$. Also, if $x$ is a periodic (mod. 1) point of period $q$ of $F$ then

$$
\rho_{F}(x)=\frac{F^{q}(x)-x}{q} \in \mathbb{Q} .
$$

The set $\left\{\rho_{F}(x): x \in \mathbb{R}\right\}=\left\{\rho_{F}(x): x \in[0,1)\right\}$ is denoted by $L_{F}$. Ito in [8] proved that $L_{F}$ is a closed interval (perhaps degenerate to a point) of $\mathbb{R}$. Thus, in the sequel $L_{F}$ will be called the rotation interval of $F$.

The rotation interval of a lifting of a circle map of degree one captures a lot of its dynamical properties and plays a fundamental role in their study (see for instance [10] and [2]).

Let $F$ be a lifting of a circle map of degree one. We define (see $[\mathbf{1}]$ )

$$
F_{u}(x)=\sup \{F(y): y \leq x\}
$$


It is not difficult to see that $F_{u}$ is non-decreasing and that it is a lifting of a circle map of degree one. Moreover, $F \leq F_{u}$.

Now we are ready to state and prove the lemma we are looking for.

Lemma 2.1. Let $f$ be a circle map and let $F$ be a lifting of $f$. Then one of the following properties hold:

(a) $f$ has o horseshoe.

(b) There exist $q \in \mathbb{N}, p \in \mathbb{Z}$ and $I=[x, x+1] \subset \mathbb{R}$ such that $\left(F^{q}-p\right)(I) \subset I$.

(c) $\operatorname{Per}(f)=f$.

Proof: If $\operatorname{deg}(f) \notin\{-1,0,1\}$ then clearly $f$ has a horseshoe. Thus, (a) holds.

Assume now that $\operatorname{deg}(f)=0$. Then, $F(x+1)=F(x)$ for all $x \in \mathbb{R}$. Hence, $F(\mathbb{R})=F([0,1])=[a, b]$. If $b \leq a+1$ then we set $q=1, p=0$ and $I=[a, a+1]$; and (b) holds. Thus, assume that $b>a+1$. Let $c \in \mathbb{R}$ be such that $F(c)=a$. Clearly $F(c+1)=a$ and there exist $d \in(c, c+1)$ such that $F(d)=b$. Set $I_{1}=e([c, d])$ and $I_{2}=e([d, c+1])$. Clearly $I_{1}$ and $I_{2}$ are arcs of $\mathbf{S}^{1}$ and $f\left(I_{1}\right)=f\left(I_{2}\right) \supset \mathrm{S}^{1} \supset I_{1} \cup I_{2}$. Thus, $f$ has a horseshoe and (a) holds.

Now we consider the case $\operatorname{deg}(f)=1$. From [10] it follows that $\operatorname{Per}(f)=\emptyset$ if and only if $L_{F}=\{\alpha\}$ with $\alpha \notin \mathbb{Q}$ and that $f$ has a horseshoe if $L_{F}$ is non-degenerate. Thus we only have to consider the case $L_{F}=\{p / q\}$ with $q \in \mathbb{N}$ and $p \in \mathbb{Z}$ relatively prime. From [5] (see also [1, Theorem 3.7.20 and its proof]) it follows that there exists $x$, a periodic (mod. 1) point of $F$ of period $q$ and rotation number $p / q$, such that $F^{i}(x)=F_{u}^{i}(x)$ for all $i \geq 0$. Set

$$
\begin{aligned}
P & =\left\{F^{i}(x)+m: \quad i=0,1,2, \ldots, q-1, m \in \mathbb{Z}\right\}= \\
& =e^{-1}\left(\left\{f^{i}(e(x)): \quad i=0,1,2, \ldots, q-1\right\}\right)= \\
& =\left\{\ldots x_{-2}, x_{-1}, x_{0}, x_{1}, x_{2}, \ldots\right\}
\end{aligned}
$$

with $\left(x_{i}, x_{i+1}\right) \cap P=\emptyset$ for all $i \in \mathbb{Z}$. Set also $G=F^{q}-p$ and $G_{u}=F_{u}^{q}-p$. Since $F_{u}$ is non-decreasing so is $G_{u}$ and, hence, $G \leq G_{u}$ because $F \leq F_{u}$. Therefore, if $y \in \mathbb{R}, z \in P$ and $y \leq z$ then

$$
G(y) \leq G_{u}(y) \leq G_{u}(z)=G(z)=z .
$$

Moreover, it is not difficult to see that $L_{G}=\{0\}$.

Set $J=\left[x_{0}, x_{0}+1\right]$. We note that $J \subset G(J)$ because $G\left(x_{i}\right)=x_{i}$ for all $i \in \mathbb{Z}$. Thus, $G^{2}(J) \subset G^{i+1}(J)$ for all $i \in \mathbb{N}$. Let $K$ be the 
closure of $\bigcup_{i=1}^{\infty} G^{i}(J)$. Clearly $K$ is a closed interval such that $J \subset K \subset$ $\left(-\infty, x_{0}+1\right]$ and $G(K) \subset K$. If $K$ is not bounded, then there exist $\bar{z} \in J$ and $m \in \mathbb{N}$ such that $G^{m}(\bar{z})<x_{0}-1$. Therefore, there exists $z \in J$ such that $G^{m}(z)=z-1$. That is, $z$ is a periodic (mod. 1) point of $G$ with rotation number $-1 / m$. This contradicts the fact that $L_{G}=\{0\}$. Thus, $K$ is of the form $\left[\alpha, x_{0}+1\right]$ with $\alpha \leq x_{0}$.

If $\alpha \in P$ then we take $I=[\alpha, \alpha+1]$. Since $\alpha+1 \in P$ we have that $G(I) \subset(-\infty, \alpha+1]$. On the other hand, $G(I) \subset G(K) \subset K$. Thus $G(I) \subset I$ and we are done.

Now assume that $\alpha \notin P$. Then there exists $i<0$ such that $\alpha \in$ $\left(x_{i}, x_{i+1}\right)$. Since $G\left(x_{i}\right)=x_{i}$ there exists a fixed point of $G$ in $\left[x_{i}, \alpha\right]$. Let $y$ be the supremum of these fixed points. Since $G(\alpha) \geq \alpha$ we see that for each $z \in[y, a]$ we have that $G(z) \geq y$. Thus the set

$$
S=\left\{y \in\left[x_{i}, \alpha\right]: G(y)=y \text { and } G(z) \geq y \text { for each } z \in[y, \alpha]\right\}
$$

is non-empty. So we set $\beta=\inf S$ and $\widetilde{K}=\left[\beta, x_{0}+1\right]$.

Since $x_{i+1} \in P$ and $x_{i+1} \leq x_{0}$ we have that $G([\beta, \alpha]) \subset\left[\beta, x_{i+1}\right] \subset \widetilde{K}$. Therefore, $G(\widetilde{K}) \subset \widetilde{K}$. Now, if $\beta=x_{i}$ we set $I=[\beta, \beta+1]$ and we proceed as above to get $G(I) \subset I$. So we may assume that $\beta \neq x_{2}$.

If $G\left(\left[x_{2}, \beta\right]\right) \subset[-\infty, \beta]$ then we shall show that $G(I) \subset I$ with $I=$ $[\beta, \beta+1]$. To see this it is enough to prove that if $z<\beta+1$ then $G(z) \leq \beta+1$ because $I \subset \widetilde{K}$ and $G(\widetilde{K}) \subset \widetilde{K}$. We shall prove first that if $z \leq \beta$ then $G(z) \leq \beta$. If $z \in\left[x_{i}, \beta\right]$ then this follows by the assumption. If $z \leq x_{i}$ then, since $x_{i} \in P$ we have that $G(z) \leq x_{i}<\beta$. Hence, since $G$ is a lifting of $f^{q}$ which has degree one, for each $z \leq \beta+1$ we have $G(z)=G(z-1)+1 \leq \beta+1$ and we are done. Thus, we may assume that there exists $t \in\left[x_{i}, \beta\right]$ such that $G(t)>\beta$.

Since $x_{i} \neq \beta$, by the definition of $\beta$, there exists $\tilde{z} \in\left[x_{i}, \beta\right]$ such that $G(\widetilde{z})<x_{i}$. If $t<\widetilde{z}$ then the intervals $[t, \tilde{z}]$ and $[\widetilde{z}, \beta]$ form a horseshoe of $G$. So $F$ has a horseshoe and (a) holds. If $\widetilde{z}<t$ then there is a fixed point of $G$ in $(\tilde{z}, t)$. Let $\gamma$ be the supremum of these fixed points. We have

$$
\begin{array}{ll}
\gamma<t<\beta & \text { and } \\
G(z) \geq \gamma & \text { for all } z \in[\gamma, t] .
\end{array}
$$

If also $G(z) \geq \gamma$ for all $z \in[t, \beta]$ then $\gamma \in S$ which contradicts the lact that $\beta=\inf S$. So, there exists $\widetilde{\gamma} \in(t, \beta)$ such that $f(\tilde{\gamma})=\gamma$. Then the intervals $[\gamma, t]$ and $[t, \tilde{\gamma}]$ form a horseshoe of $G$. This ends the proof of the lemma in the case when $f$ has degree one.

Finally assume that $\operatorname{deg}(f)=-1$. By the definition of degree of a map, $f$ has a fixed point. Thus, $\operatorname{Per}(f) \neq 0$. Let us consider $f^{2}$. It has 
degree 1 and $F^{2}$ as a lifting. Thus, in view of the degree one case, either $f^{2}$ has a horseshoe or there exist $q \in \mathbb{N}, p \in \mathbb{Z}$ and $I=[x, x+1]$ such that $\left(\left(F^{2}\right)^{q}-p\right)(I) \subset I$. Hence, either $f$ has a horseshoe or $\left(F^{2 q}-p\right)(I) \subset I$.

Remark 2.2. From the proof of the above lemma it follows that if $f$ is a circle map satisfying (b) of Lemma 2.1 , then $\operatorname{deg}(f) \in\{-1,0,1\}$.

\section{Proof of Theorem 1.2.}

We shall start with some technical lemmas on interval maps.

Lemma 3.1. Let $f$ be o continuous map of the interval I into itself having a horseshoe. Then, $f$ has a horseshoe in $\operatorname{Int}(I)$.

Proof: Since $f$ has a horseshoe there exist $n \in \mathbb{N}$ and $I_{1}, I_{2} \subset I$, two closed intervals with pairwise disjoint interiors, such that

$$
I_{1} \cup I_{2} \subset f^{n}\left(I_{1}\right) \quad \text { and } \quad I_{1} \cup I_{2} \subset f^{n}\left(I_{2}\right)
$$

Then, there exist $J_{1}^{1}, J_{2}^{1} \subset I_{1}$ and $J_{1}^{2}, J_{2}^{2} \subset I_{2}$, closed intervals with pairwise disjoint interiors such that

$$
J_{1}^{1} \cup J_{2}^{1} \cup J_{1}^{2} \cup J_{2}^{2} \subset f^{2 n}\left(J_{j}^{i}\right)
$$

with $i, j \in\{1,2\}$. Clearly, two of these intervals are contained in $\operatorname{Int}(I)$. Then, $f$ has a horseshoe in $\operatorname{Int}(I)$.

Lemma 3.2. Let $f$ be a continuous map of the interval $I=[a, a+1]$ into itself such that $f(a+1)=f(a)+d$ with $d \in\{-1,0,1\}$. Assume that $\omega_{x}(f)$ is a simple set for any $x \in I$ and that $a \in \omega_{x}(f)$ and $a+1 \in \omega_{y}(f)$ for some $x, y \in I$. Then the following statements hold:

(a) If $\omega_{x}(f) \neq \omega_{y}(f)$ then $\omega_{x}(f)$ and $\omega_{y}(f)$ are fixed points of $f$.

(b) If $\omega_{x}(f)=\omega_{y}(f)$ then $\omega_{x}(f)$ is a periodic orbit of period 2 of $f$.

Proof: It will be divided into several cases.

Case 1: $d=0$. We shall prove that $\{a, a+1\} \not \subset \omega(f)$ and thus the lemma holds. In view of Theorem A and the fact that for an interval map the chain recurrent set is a union of simple sets if and only if each omega limit set is a simple set, it is cnough to show that if $\{a, a+1\} \subset \omega(f)$ then $f$ has a horseshoe.

If $f(a+1)=f(a)=a$ then, since $a+I \in \omega_{y}(f)$ and $\omega_{y}(f)$ is invariant, it follows that there exists $z \in I$ such that $f(z)=a+1$. Thus, $[a, z]$ and $[z, a+1]$ form a horseshoe for $f$. 
If $f(a+1)=f(a)=a+1$ we proceed in a similar way to obtain a horseshoe for $f$.

Assume now that $a<f(a)=f(a+1)<a+1$. As before, there exist $z, z^{\prime} \in I$ such that $f(z)=a$ and $f\left(z^{\prime}\right)=a+1$. Moreover, there exists $t$, a fixed point of $f$ between $z$ and $z^{\prime}$.

If $z<z^{\prime}$ and $f(a) \geq t$ then the intervals $[a, z]$ and $[z, t]$ form a horseshoe of $f$. If $z<z^{\prime}$ and $f(a)<t$ then the horseshoe of $f$ is given by $\left[t, z^{\prime}\right]$ and $\left[z^{\prime}, a+1\right]$. Thus, if $z<z^{\prime}$ we are done.

If $z^{\prime}<z$ and $f(a) \geq t$ we set $A=\left[a, z^{\prime}\right], B=\left[z^{\prime}, t\right]$ and $C=[t, z]$. Then we have $f(A) \supset C, f(B) \supset C$ and $f(C) \supset A \cup B$. Hence,

$$
f^{2}(A) \supset A \cup B \text { and } f^{2}(B) \supset A \cup B .
$$

Thus $A$ and $B$ form a horseshoe of $f$. If $z^{\prime}<z$ but $f(a)<t$ then, in a similar way we can see that $[t, z]$ and $[z, a+1]$ form a horseshoe of $f$.

Case 2: $d=1$. We have that $f(a)=a$ and $f(a+1)=a+1$. Thus, $\omega_{x}(f)$ and $\omega_{y}(f)$ are simple sets which contain a fixed point. In view of Remark 1.1 we obtain that $\omega_{x}(f)=\{a\}$ and $\omega_{y}(f)=\{a+1\}$.

Case 3: $d=-1$. We have that $\{a, a+1\}$ is now a periodic orbit of $f$ of period 2. Thus, again by Remark 1.1 we see that $\omega_{x}(f)=\omega_{y}(f)=$ $\{a, a+1\}$.

Let now $f$ be a circle map and let $F$ be a lifting of $f$. We say that $f \in \mathcal{A}$ if there exist $x \in \mathbb{R}$ and $p \in \mathbb{Z}$ such that $(F-p)([x, x+1]) \subset[x, x+1]$. That is, if $F$ satisfies (b) of Lemma 2.1 with $q=1$. From Remark 2.2 we note that $\operatorname{deg}(f) \in\{-1,0,1\}$.

Lemma 3.3. Each map $f \in \mathcal{A}$ has one and only one from the following two properties:

(a) $f$ has a horseshoe.

(b) $\omega_{x}(f)$ is a simple set for each $x \in \mathbf{S}^{1}$.

Proof: Let $F$ be a lifting of $f$. Since $f \in \mathcal{A}$ there exist $x \in \mathbb{R}$ and $p \in \mathbb{Z}$ such that $(F-p)(\mid x, x+1]) \subset[x, x+1]$. In view of Theorem $\mathrm{A}$, Lemmas 3.1 and 3.2 and the fact that the chain recurrent set of an interval map is union of simple sets if and only if each omega limit set is a simple set we get that $(F-p)$ has one and only one from the following two properties.

(i) $(F-p)$ has a horseshoe in $(x, x+1)$.

(ii) $\omega_{y}(F-p)$ is a simple set for each $y \in[x, x+1]$ and if $x \in \omega_{x_{1}}(F-p)$ and $x+1 \in \omega_{x_{2}}(F-p)$ for some $x_{2}, x_{2} \in[x, x+1]$ then $\omega_{x_{1}}(F-p)$ 
and $\omega_{x_{2}}(f-p)$ are fixed points of $(F-p)$ when $\omega_{x_{1}}(F-p)=$ $\omega_{x_{2}}(F-p)$ and $\omega_{x_{1}}(F-p)$ is a periodic orbit of period 2 when $\omega_{x_{1}}(F-p) \neq \omega_{x_{2}}(F-p)$.

Since $(F-p)$ is also a lifting of $f$ and $e:(x, x+1) \longrightarrow \mathbf{S}^{1} \backslash\{e(x)\}$ is a homeomorphism we get the desired conclusion.

Now we are ready to prove the main result of this paper.

Proof of Theorem 1.2: It follows straightforwardly from Lemmas 2.1 and 3.3 and from the fact that if $f$ satisfies (a) or (b) of Lemma 2.1 then $\operatorname{Per}(f) \neq \emptyset$.

\section{A geometric view-point of Condition (b) of Theorem 1.2.}

Throughout this section we will assume that $f$ is a circle map satisfying Condition (b) of Theorem 1.2. We shall try to explain this condition in terms of the graph of the map and the chain recurrent set of $f$.

From Remark 2.2 we have that $\operatorname{deg}(f) \in\{-1,0,1\}$. If $\operatorname{deg}(f) \in$ $\{-1,0\}$, by looking at the proof of Theorem 1.2 , we can prove that the situation for the chain recurrent set of $f$ is the same as in the case of interval maps. That is, the chain recurrent set of $f$ is the union of all simple sets.

Now assume that $\operatorname{deg}(f)=1$. Also from the proof of Lemma 2.1 and from the fact that a circle map satisfies one and only one from the conditions of Theorem 1.2 we see that if $F$ is a lifting of $f$ then $L_{F}=\{p / q\}$ with $q \in \mathbb{N}$ and $p \in \mathbb{Z}$ relatively prime. By using the notation from the proof of Lemma 2.1 we see that there exists a periodic (mod. 1) point $\beta$ of $F$ with rotation number $p / q$ such that

$$
\left(F^{q}-p\right)([\beta, \beta+1]) \subset[\beta, \beta+1] .
$$

If we set

$$
\begin{aligned}
Q & =\left\{F^{i}(\beta)+m: i=0,1,2, \ldots, q-1, m \in \mathbb{Z}\right\}= \\
& =e^{-1}\left(\left\{f^{i}(e(\beta)): i=0,1,2, \ldots, q-1\right\}\right)= \\
& =\left\{\ldots \beta_{-2}, \beta_{-1}, \beta_{0}, \beta_{1}, \beta_{2} \ldots\right\}
\end{aligned}
$$

then it is not difficult to prove that $\left(F^{q}-p\right)\left(\left[\beta_{i}, \beta_{i+1}\right]\right) \subset\left[\beta_{i}, \beta_{i+1}\right]$ for all $i \in \mathbb{Z}$ and that for each $i, j \in \mathbb{Z}$ the diagram 


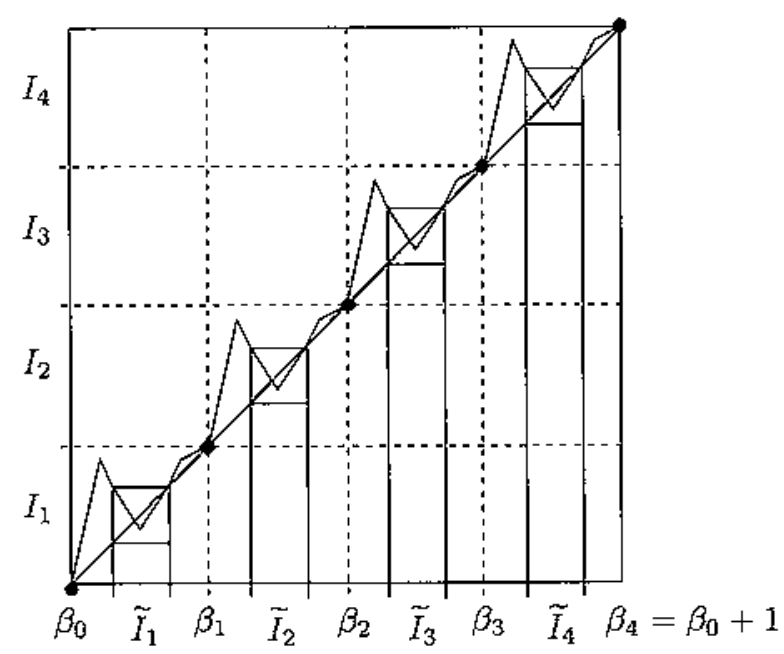

Figure 1: The typical situation of a chain recurrent set of a circle map $f$ of degrec one. Fach $\beta_{i}$ is a periodic (mod. 1) point of $F$ of period 4 and rotation number $p / 4$ for some $p \in \mathbb{Z}$. The picture shows the graph of the map $F^{4}-p$. Here $R_{i} \subset \widetilde{I}_{i} \cup\left\{\beta_{i}\right\}$ and $T_{i} \subset I_{i} \backslash\left(\tilde{I}_{i} \cup\left\{\beta_{i}\right\}\right)$.

$$
\begin{array}{cc}
{\left[\beta_{i}, \beta_{i+1}\right] \stackrel{\left.G\right|_{\left[\beta_{i}, \beta_{i+1}\right]} ^{\longrightarrow}}{\longrightarrow}\left[\beta_{i}, \beta_{i+1}\right]} \\
\left.h_{i j}\right\rfloor \\
{\left[\beta_{j}, \beta_{j+1}\right] \underset{\left.G\right|_{\left[\beta_{j}, \beta_{j+1}\right]} ^{\longrightarrow}}{\stackrel{h_{i j}}{\longrightarrow}}\left[\beta_{j}, \beta_{j+1}\right]}
\end{array}
$$

commutes, where $h_{i j}$ is the unique bijective affine map from $\left[\beta_{i}, \beta_{i+1}\right]$ to $\left[\beta_{j}, \beta_{j+1}\right]$.

It is also easy to see (see [1] for instance) that $\beta_{i+q}=\beta_{i}+1$ and $F\left(\beta_{i}\right)=\beta_{i+p}$ for each $i \in \mathbb{Z}$. Thus, if we define $I_{i}=e\left(\left[\beta_{i}, \beta_{i+1}\right)\right)$ for $i=0,1,2, \ldots, q-1$ we get that $f\left(I_{i}\right)=Y_{i+p(\bmod q)}$ and $\left.f^{q}\right|_{I_{i}}$ is conjugate to $\left.f^{q}\right|_{I_{j}}$ for each $i, j \in\{0,1,2, \ldots, q-1\}$.

Let now $C$ denote the chain recurrent set of $f$ and set $C_{i}=C \cap I_{i}$ for $i=0,1, \ldots, q-1$. Since $C$ is invariant we have that $f\left(C_{i}\right)=C_{i+p(\bmod q)}$ for each $i \in\{0,1,2, \ldots, q-1\}$. That is, the chain recurrent set is formed 
by $q$ "rotating" disjoint pieces. Moreover, $C_{i}=C R\left(f^{q}\right) \cap I_{i}$ and $\left.f^{q}\right|_{C_{2}}$ is conjugate to $\left.f^{q}\right|_{C_{j}}$ for each $i, j \in\{0,1,2, \ldots, q-1\}$. To end the description of the dynamics of $f$ on $C$ we still need to describe the dynamics of $f^{q}$ on the sets $C_{i}$. Each of these sets $C_{i}$ can be decomposed in a union of two more sets: $R_{i}$ and $T_{i}$. In general the set $T_{i}$ can be empty while $R_{i}$ it is not. The dynamics of $\left.f^{q}\right|_{R_{\mathrm{i}}}$ is the one of a simple interval map on its chain recurrent set. That is, $R_{i}$ is the union of all simple sets of $\left.f^{q}\right|_{I_{i}}$. On the other hand, $\left.f\right|_{T_{t}}$ is a monotone map. A typical situation where $T_{i} \neq \emptyset$ is illustrated in Figure 1.

\section{References}

1. Ll. Alsedì, I. Llibre and M. Misilrewicz, "Combinatorial dynamics and entropy in dimension one," Advanced Series on Nonlinear Dynamics 5, World Scientific, Singapore, 1993.

2. Ll. Alsedà, J. Llibre, F. Mañosas and M. Misiurewicz, Lower bounds of the topological entropy for continuous maps of the circle of degree one, Nonlinearity 1 (1988), 463-479.

3. J. AUSLANDER AND Y. KATZNELSON, Continuous maps of the circle without periodic points, Israel J. Math. 32 (1979), 375381.

4. L. S. BlOCK AND W. A. COPPEL, "Dynamics in one dimension," Lecture Notes in Math. 1513, Springer, Berlin, 1992.

5. A. Chenciner, J.-M. Gambaudo and C. Tresser, Une remaique sur la structure des endomorphismes de degré 1 du cercle, $C$. R. Acad. Sci. Paris, Sér. I Math. 299 (1984), 145-148.

6. V. V. FEDORENKO, Dynamics of interval maps with zero topological entropy on the set of chain recurrent points (in Russian), Dynamical systems and nonlinear phenomena, Inst. Mat. Akad. Nauk, Ukrain., Kiev (1989).

7. V. V. Fedorenko, Simple dynamics of the interval maps, Preprint 186, Centre de Recerca Matemàtica (1993).

8. R. ITo, Rotation sets are closed, Math. Proc. Camb. Phit. Soc. 89 (1981), 107-111.

9. M. Misiurewicz, Horseshoes for mappings of an interval, Bull. Acad. Pol. Sci., Sér. Sci. Math. 27 (1979), 167-169.

10. M. Misiurewicz, Periodic points of maps of degree one of a circle, Ergod. Th. \& Dynam. Sys. 2 (1982), 221227.

11. M. Misiurewicz, Twist sets for maps of the circle, Ergod. Th. \& Dynam. Sys, 4 (1984), 391-404. 
12. A. N. SHARKovsKII, Co-existence of the cycles of a continuous mapping of the line into itself (in Russian), Ukrain. Math. $Z$ h. 16 (1) (1964), 61-71.

13. A. N. ShaRKovSKIĬ, On cycles and the structure of a continuous mapping (in Russian), Ukrain. Math. Zh. 17 (3) (1965), 104-111.

Lluîs Alsedà:

Departament de Matemátiques Universitat Autonoma de Barcelona 08193 Bellaterra (Barcelona)

SPAIN
Vladimir Fedorenko:

Institute of Mathematics

Ukrainian Academy of Sciences

Repin str. 3

$252601 \mathrm{Kiev}-4$

UKRAINE

Primera versió rẹbuda el 17 de Novembre de 1992, darrera versió rebuda el 19 d'Abril de 1993 